the literature - while also acting as a powerful deterrent to would be plagiarists.

In the process, editors and publishers must remember that plagiarism comes in many varieties and degrees of severity, and that responses should be proportionate. For example, past studies suggest that self-plagiarism, in which a researcher copies his or her own words from a published paper, is far more common than plagiarism of the work of others. Arguably, self-plagiarism can sometimes be justified, as when a researcher is bringing similar ideas before readers of journals in a different field. All plagiarism can also involve honest errors or mitigating circumstances, such as a scientist with a poor command of English paraphrasing some sentences of the introduction from similar work.

Such examples underscore that plagiarism-detection software is an aid to, not a substitute for, human judgement. One rule of thumb used by Nature journals and others in considering an article's degree of similarity to past articles - in particular, for small amounts of self-plagiarism in review articles - is whether the paper is otherwise of sufficient originality and interest.

Nature Publishing Group is a member of CrossCheck and has been testing the service on submissions to its own journals. It has noted only trace levels of plagiarism in research articles, which are spot-checked, and often in only the supplementary methods. Plagiarism has been more common in submitted reviews, all of which are tested. This is particularly true in clinical reviews, although the rates are still far below the $1 \%$ mark, and in most instances concerned some level of self-plagiarism.

Although the ability to detect plagiarism is a welcome advance, addressing the problem at its source remains the key issue. More and more learned societies, research institutions and journals have in recent years adopted comprehensive ethical guidelines on plagiarism, many of which carefully distinguish between different levels of severity. It is crucial that research organizations in all countries, and particularly the mentors of young researchers, instil in their scientists the accepted norms of the international scientific community when it comes to plagiarism and publication ethics.

\section{The needs of the few}

\section{Developing drugs for rare diseases is a challenge that requires new regulatory flexibility.}

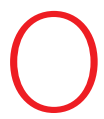
n 29 June, Timothy Coté, head of the Office of Orphan Products Development at the US Food and Drug Administration (FDA), concisely summed up the agency's policies with respect to the approval of drugs and other medicinal products for rare diseases: "No policy at all."

The irony of this assessment is that the United States has long been a leader in stimulating the development of therapies for rare diseases. Congress passed the Orphan Drug Act in 1983 in an attempt to deal with the unique commercial and regulatory challenges posed by 'orphan' diseases, defined as those that affect fewer than 200,000 Americans. For industry, there is little appeal in pursuing a drug that will be required by only a small number of patients. For regulators accustomed to the clinical trials typically performed for common diseases, it can be difficult to ascertain the safety of a drug that, by necessity, can be tested in only a tiny cohort of patients.

The act aimed to incentivize orphan-drug development by rewarding drug-makers with a seven-year period of market exclusivity for such compounds. The FDA also created the Office of Orphan Products Development to shepherd companies through the approval process. Ten years later, Japan enacted similar legislation, and Europe followed suit in 2000 .

In many ways the act was a success. In the decade before its passage, the FDA approved fewer than a dozen drugs for rare diseases; since then, the agency has approved 358 . Nevertheless, the vast majority of the 7,000 known rare diseases remain without treatment. And, as Coté was explaining last week at the inaugural meeting of the FDA's new expert panel on orphan diseases, the agency still has no policy guiding how it evaluates possible treatments for a rare disease.
It is time for the FDA to develop one. The ranks of orphan diseases are growing. Better understanding of common ailments - for example, through genome sequencing - is shattering old classification schemes, fragmenting many 'common' diseases into smaller subtypes. The medical landscape will soon be crowded with 'orphans'.

This means that the FDA will be seeing more applications bearing data from small clinical trials, thrusting regulators into the uncomfortable position of ascertaining safety and efficacy with less than optimal data. Classical gold-standard, placebo-controlled studies force researchers to divide their already tiny experimental cohort in half - one half that receives the experimental drug, the other a placebo. And because these diseases are often fatal (of those afflicted with one of the 350 most common rare diseases, $27 \%$ will not see their first birthday), patients are understandably loath to spend much time receiving a placebo.

As a result, the FDA will need to allow more flexibility in clinicaltrial design. In some cases this may mean a short placebo-controlled study that moves rapidly into an open-label trial, in which both researchers and patients know what is being administered. In other cases it may mean abandoning placebo controls altogether. Furthermore, post-marketing studies to monitor safety and efficacy of drugs after approval may have to be done with smaller sample sizes than are normally required. The FDA could also learn from Europe, which has carved out an 'exceptional circumstances' pathway to approval for therapies for which full, gold-standard clinical-trial data are not available.

All of these issues will be under consideration as the agency's new expert panel prepares an advisory report, due to be released in September. There are signs that it will fall on receptive ears: in remarks made before the Senate in March, FDA commissioner Margaret Hamburg expressed a commitment to finding new solutions to the problem of rare diseases. And two large pharmaceutical companies, GlaxoSmithKline and Pfizer, have recently announced new research divisions dedicated to orphan diseases. The present momentum should not be allowed to fail. 